\title{
Semiótica do discurso científico: um estudo sobre novas perspectivas para a análise documental de conteúdo
}

Semiótica del discurso científico: un estudio sobre nuevas perspectivas para el análisis documental del contenido

Semiotic of scientific discourse: a study on new perspectives for documentary content analysis

\author{
Roberta Caroline Vesú Alves (1), João Batista Ernesto de MoRAes (2), Carlos Cândido de AlmeidA (3) \\ (1) Faculdade de Filosofia e Ciências-Unesp, Av. Higyno Muzzi Filho, 737, Marília - São Paulo - Brasil - CEP:17525-900, \\ robertavesu@gmail.com (2) jota@marilia.unesp.br (3) carlosalmeida@marilia.unesp.br
}

\begin{abstract}
Resumen
Se realiza un estudio exploratorio a partir de la revisión de la literatura sobre la producción de sentido en el discurso científico desde la perspectiva de la semiótica greimasiana, que ofrece un marco metodológico general para el análisis de los diferentes tipos de textos y discursos. Se muestra como la semiótica de Greimas y el análisis del recorrido generativo de sentido permiten la identificación de la tematicidad (aboutness ) de los documentos científicos durante el análisis documental del contenido.
\end{abstract}

Palabras clave: Semiótica greimasiana. Discurso científico. Recorrido generativo de sentido. Análisis documental de contenido.

\section{Introdução}

A Análise Documental de Conteúdo identifica e representa o conteúdo para recuperação de documentos. Nesse sentido, o aboutness de textos narrativos ficcionais pode ser identificado por meio da análise da estrutura textual elucidada pelo Percurso Gerativo de Sentido, teoria da área de Análise do Discurso e proveniente da Semiótica Francesa de Greimas (1917-1992).

Dentre as diversas linhas semióticas, a Semiótica Greimasiana parece a que mais elementos fornece aos estudos textuais, bem como a identificação de conceitos. Diferentemente da abordagem de Charles Peirce, com orientação lógica, e da abordagem clássica de Ferdinand de Saussure, cujo objetivo foi lançar as bases estruturais da Semiologia francesa.

Nesse âmbito, a Semiótica Greimasiana desenvolveu também estudos que explicam a produção de sentido de diferentes tipos de discursos independentes de sua manifestação, como o científico. Ainda, considera textos verbais e nãoverbais, além do contexto social do discurso.

\begin{abstract}
Greimas Semiotic offers procedures for the analysis of different types of texts and discourses. For this reason, this study aimed to analyze the production of sense from scientific discourse, explained by Greimas Semiotics and Generative Sense Course, seeking to develop a new perspective on the identification of aboutness in Documentary Content Analysis and Information Science. The method used was exploratory and bibliographic review. Results show that the analysis of scientific discourse contributes to the identification of aboutness in Documentary Content Analysis, using Greimas Semiotics and Generative Sense Course.
\end{abstract}

Keywords: Greimas Semiotics. Scientific discourse. Generative sense course. Documentary content analysis. Aboutness.

Greimas contribuiu, sobretudo, para propor uma lógica no exame das ações dos agentes no interior de uma narrativa, buscando identificar, seguindo a linha estruturalista, as condições subjacentes do sistema que formam o discurso. Por isso, o objetivo deste estudo consistiu em analisar o discurso científico por meio da Semiótica Greimasiana e Percurso Gerativo de Sentido, visando também ao desenvolvimento de uma nova perspectiva de identificação do aboutness de documentos com discursos científicos em Análise Documental de Conteúdo.

Para isso, a pesquisa foi desenvolvida pelo método de estudo exploratório e revisão bibliográfica para o embasamento teórico. Como resultado, verificou-se que a teoria da Semiótica Greimasiana contribui para compreensão do sentido de textos científicos, consequentemente, para a identificação do aboutness em Análise Documental de Conteúdo. 


\section{Análise Documental de Conteúdo e Aboutness}

A Análise Documental de Conteúdo desenvolve os procedimentos para a representação de documentos de forma distinta da original, para fins de recuperação (Gardin et al., 1981). Esses procedimentos iniciam com a etapa de análise do documento, com a identificação de conceitos para posterior tradução e representação (Guimarães, Moraes e Guarido, 2007).

Nesse contexto, o aboutness é considerado para esta pesquisa como equivalente aos conceitos identificados. Segundo Beghtol (1986), os documentos contêm aboutness, (assunto intrínseco ao documento, relativamente permanente), e meaning (que é mutável devido ao contexto, local, momento histórico, interesse etc.).

Para identificação do aboutness é importante observar a relação explícita entre as entidades e suas representações, garantindo que não ocorram equívocos ou desvios na representação (Moraes, 2011). Também é importante que uma expressão faça sentido e seja um conteúdo relevante, obtendo seu sentido a partir da relação entre os termos do texto (Moraes, 2011).

Por isso, para identificar o conteúdo do texto, segundo a relação entre seus termos, são utilizadas as regras de criação desse texto, que explicam a produção de sentido. No caso do texto de ficção, o sentido é compreendido com a análise do Percurso Gerativo de Sentido, importante para a identificação do aboutness em Análise Documental de Conteúdo. Esse Percurso contém três níveis, cada um com sintaxe e semântica. Segundo Barros (2008), o nível profundo e abstrato consiste na relação de oposição entre termos em quadrado semiótico. $O$ nível narrativo contém o enunciado elementar de relação entre sujeito e objeto, o programa narrativo (enunciado de fazer que rege um enunciado de estado), além de percurso e esquema narrativo, e as modalizações do ser e do fazer. Este, também relacionado às fases de manipulação, competência, performance e sanção (Alves, 2008; Antonio; Moraes, 2010; Fiorin, 2008, 1999; Tatit, 2003). O nível discursivo apresenta as projeções da enunciação, a tematização e figurativização, além de isotopias (Barros, 2008; Greimas, 1973).

Segundo Moraes, Damazo e Lara (2008), o tema de nível profundo e principal do texto literário está concentrado nas fases de manipulação e sanção. Assim, é possível construir o quadrado semiótico com termos opostos, que não estão explícitos e que correspondem ao aboutness (Alves, 2008; Moraes, 2011).
Greimas considera a estrutura geral de significação inerente aos textos verbais e não-verbais diversos e passíveis de descrição semiótica, desenvolvendo também sua abordagem sintáxica do texto integral (Antonio e Moraes 2010). Por isso, entre outros aspectos, estabelece as bases apara o Percurso Gerativo de Sentido, que elucida os elementos de sintaxe e semântica dos textos, de acordo com patamares que levam à compreensão de elementos simples e complexos para a identificação do tema do texto, também para fins de Análise Documental de Conteúdo (Antonio e Moraes 2010).

Nesse sentido, foram analisadas as contribuições da Semiótica Greimasiana para a identificação do aboutness do texto científico.

\section{Semiótica do Discurso Científico}

Greimas, na construção de seu modelo, foi influenciado pela obra de Propp sobre a morfologia do conto popular russo, o que está na base do modelo actancial que transcende toda análise semiótica. Nesse sentido, sua Semiótica, mais que uma ciência, uma teoria da significação, procura analisar de maneira macroestrutural diversas tipologias discursivas.

A Semiótica do discurso científico, segundo Greimas e Landowski (1979, p. 12), "[...] tem em vista unicamente a explicitação das formas discursivas e de sua tipologia", sendo que o estudo da tipologia não prevê uma regulamentação dos discursos, em "dever-ser", mas sim a análise do que é, o "ser", e a maneira como os discursosobjeto instauram suas normas. Para isso, os autores reuniram estudos sobre discursos que representam as pesquisas em Ciências Humanas e Sociais, identificando três grandes tipos de discursos em pesquisa (Greimas e Landowski, 1979, p. 15).:

1. Discursos em busca de certezas científicas;

2. Interrogações sobre o próprio sentido da pesquisa;

3. Discursos de interpretação.

De modo sucinto, segundo os autores, para análise desses discursos é considerado, entre outros fatores, o discurso cognitivo reconhecido em uma pesquisa como uma narrativa, que corresponde à ideia de uma simples transformação de estado. Assim, apresenta um primeiro estado de não-saber e a passagem para o estado final, um saber, com a conjunção ao objeto de valor (Greimas, 1976). Nesse sentido, o sujeito é dotado de competência cognitiva. Esses procedimentos de narrativização do discurso cognitivo tomados como objeto são importantes dimensões de análise, pois (Greimas e Landowski, 1979, p. 21), 
[...] a organização do discurso cognitivo em forma de programas narrativos, que vai fornecer-nos o meio de imaginar seus principais níveis de articulação.

Dessa forma, ainda segundo os autores, em nível profundo devem existir duas formas de organização do conteúdo, uma relacionada ao estágio anterior à narrativa (busca do saber) e outra de transformação, sendo que no plano de conteúdo a transformação modal ocorre do nãosaber para o saber (Greimas e Landowski, 1979). Por isso, pode ser entendido que as fases do nível narrativo que compreendem o nãosaber e o saber correspondem, respectivamente, a manipulação e a sanção do nível narrativo do Percurso Gerativo de Sentido. Sendo que, de modo geral, o estado inicial de não-saber sobre algo necessariamente implica na busca do saber (manipulação); a competência cognitiva é o conhecimento que colabora para a busca do saber; a performance está no desenvolvimento da mudança de estado; e a sanção apresenta o estado final do saber alcançado.

Por isso, a análise da produção de sentido do discurso científico, embasada na Semiótica Greimasiana também necessita de aspectos teóricos do Percurso Gerativo de Sentido.

Além disso, vale destacar que a relação entre as dimensões cognitiva e pragmática norteia essa análise. Os programas narrativos constituem a dimensão pragmática e correspondem (Greimas e Courtés, 2008, p. 379)

[...] às descrições, que ali são feitas, dos comportamentos somáticos significantes, organizados em programas e recebidos pelo enunciatário como "acontecimentos", independentemente de sua eventual utilização no nível do saber: os objetos pragmáticos são reconhecíveis como valores descritivos [...], em oposição aos valores modais.

A dimensão pragmática mostra a relação de dependência entre os actantes. Também, essa dimensão é referente interno à dimensão cognitiva do discurso, que é desenvolvida "[...] paralelamente ao aumento do saber (como atividade cognitiva) atribuído aos sujeitos instalados no discurso." (Greimas e Courtés, 2008, p. 64). Mas, a relação contrária não ocorre, ou seja, a dimensão cognitiva é hierarquicamente superior à pragmática, estabelecendo o sentido do que é enunciado pelo pesquisador.

A partir do entendimento da produção de sentido do discurso científico foi desenvolvida a análise voltada à Ciência da Informação, identificando o tema principal de nível profundo (aboutness) conforme a Análise Documental de Conteúdo, Semiótica Greimasiana e Percurso Gerativo de Sentido.
Para demonstrar a análise e identificação do aboutness do texto científico foi utilizado um capítulo do livro de Jouve (2002), O que é a leitura?. Verificou-se o discurso cognitivo com base na dimensão pragmática, que apresenta os programas narrativos, principalmente, sobre as fases de manipulação e sanção.

Segundo Greimas (1976, 1979), o discurso científico apresenta uma "aventura cognitiva" que obedece à forma da busca do objeto de valor, este consiste em um certo saber que se procura adquirir. Portanto (ibidem, p. 41),

Sendo o objeto-saber o objetivo do discurso, é evidente que o estado inicial de que parte a busca é um estado de não-saber: a narrativa científica define-se, então, como a transformação de um /não-saber/ em um /saber/.

A dimensão pragmática, segundo Barros (2008), contém seus programas narrativos ( $P N)$, que seguem o modelo: $P N=F\left[S_{1} \rightarrow\left(S_{2} \wedge O_{v}\right)\right](F$ função/fazer; $\rightarrow$ transformação; $S_{1}$ sujeito do fazer; $S_{2}$ sujeito do estado; $\wedge$ conjunção; $v$ disjunção; $\mathrm{O}_{v}$ objeto de valor). Como exemplo da fase de manipulação tem-se a dúvida: "O que é a leitura?" (Jouve, 2002, p. 17). O programa narrativo desta fase pode ser descrito como:

$\mathrm{PN} 1=\mathrm{F}\left[\mathrm{S}_{1}\right.$ dúvida $\rightarrow\left(\mathrm{S}_{2}\right.$ leitura $\vee \mathrm{O}_{\mathrm{v}}$ o que é $\left.)\right]$

A dúvida ou problema apresentado estabelece um estado inicial de disjunção com o conceito de leitura (o que é). A partir disso, o autor apresenta um estado de não-saber o que é a leitura, posteriormente, passa para o outro estado de saber algo sobre leitura, descrevendo a seu modo este conceito.

Alguns exemplos do estado final do saber em dimensão pragmática do texto de Jouve (2002) estão nos quadros abaixo, consequentemente, contém a sanção e seus programas narrativos:

Um processo neurofisiológico. A leitura é antes de mais nada um ato concreto, observável, que recorre a faculdades definidas do ser humano. Com efeito, nenhuma leitura é possivel sem um funcionamento do aparelho visual e de diferentes funções do cérebro. Ler é anteriormente a qualquer análise do conteúdo, uma operação de percepção, de identificação e de memorização de signos. (Jouve, 2002, p. 17).

$\mathrm{PN} 2=\mathrm{F}\left[\mathrm{S}_{1}\right.$ saber $\rightarrow\left(\mathrm{S}_{2}\right.$ leitura $\wedge \mathrm{O}_{\vee}$ processo neurofisiológico)]

$\mathrm{PN} 3=\mathrm{F}\left[\mathrm{S}_{1}\right.$ saber $\rightarrow\left(\mathrm{S}_{2}\right.$ leitura $\wedge \mathrm{O}_{v}$ ato concreto, observável, que recorre a faculdades definidas do ser humano)]

$\mathrm{PN} 4=\mathrm{F}\left[\mathrm{S}_{1}\right.$ saber $\rightarrow\left(\mathrm{S}_{2}\right.$ leitura $\wedge \mathrm{O}_{v}$ possivel com o funcionamento do aparelho visual e de diferentes funções do cérebro)]

$\mathrm{PN5}=\mathrm{F}\left[\mathrm{S}_{1}\right.$ saber $\rightarrow\left(\mathrm{S}_{2}\right.$ leitura $\wedge \mathrm{O}_{v}$ operação de percepção, de identificação e de memorização de signos)] 
Quadro I. Leitura: processo neurofisiológico

Um processo cognitivo. [...] a leitura solicita competência. O texto coloca em jogo um saber mínimo que o leitor deve possuir se quiser rosseguir a leitura. (Jouve, 2002, p. 18).

$\mathrm{PN6}=\mathrm{F}\left[\mathrm{S}_{1}\right.$ saber $\rightarrow\left(\mathrm{S}_{2}\right.$ leitura $\wedge \mathrm{O}_{\vee}$ processo cognitivo $\left.)\right]$ $\mathrm{PN7}=\mathrm{F}\left[\mathrm{S}_{1}\right.$ saber $\rightarrow\left(\mathrm{S}_{2}\right.$ leitura $\wedge \mathrm{O}_{\vee}$ solicita competência)]

$\mathrm{PN} 8=\mathrm{F}\left[\mathrm{S}_{1}\right.$ saber $\rightarrow\left(\mathrm{S}_{2}\right.$ leitura $\wedge \mathrm{O}_{v}$ um saber mínimo que o leitor deve possuir)]

Quadro II. Leitura: processo cognitivo

"Um processo argumentativo" (Jouve, 2002, p. 21).

Qualquer que seja o tipo de texto, o leitor, de forma mais ou menos nítida, é sempre interpelado. Trata-se para ele de assumir ou não para si próprio a argumentação desenvolvida. (Jouve, 2002, p. 22)

$\mathrm{PN} 10=\mathrm{F}\left[\mathrm{S}_{1}\right.$ saber $\rightarrow\left(\mathrm{S}_{2}\right.$ leitura $\wedge \mathrm{O}_{v}$ processo argumentativo)]

$\mathrm{PN} 11=\mathrm{F}\left[\mathrm{S}_{1}\right.$ saber $\rightarrow\left(\mathrm{S}_{2}\right.$ leitura $\wedge \mathrm{O}_{\vee}$ o leitor, de forma mais ou menos nitida, é sempre interpelado)]

$\mathrm{PN} 12=\mathrm{F}\left[\mathrm{S}_{1}\right.$ saber $\rightarrow\left(\mathrm{S}_{2}\right.$ leitura $\wedge \mathrm{O}_{\mathrm{v}}$ assumir ou não para si próprio a argumentação desenvolvida)]

Quadro III. Leitura: processo argumentativo

Um processo simbólico. O sentido que se tira da leitura (reagindo em face a história, dos argumentos propostos, do jogo entre os pontos de vista) vai se instalar imediatamente no contexto cultural onde cada leitor evolui. Toda leitura interage com a cultura e os esquemas dominantes de um meio e de uma época. A leitura afirma sua dimensão simbólica agindo nos modelos do imaginário coletivo quer os recuse quer os aceite. (Jouve, 2002, p. 22).

$\mathrm{PN13}=\mathrm{F}\left[\mathrm{S}_{1}\right.$ saber $\rightarrow\left(\mathrm{S}_{2}\right.$ leitura $\wedge \mathrm{O}_{\vee}$ processo simbólico)]

$\mathrm{PN} 14=\mathrm{F}\left[\mathrm{S}_{1}\right.$ saber $\rightarrow\left(\mathrm{S}_{2}\right.$ leitura $\wedge \mathrm{O}_{v}$ sentido que vai se instalar imediatamente no contexto cultural onde cada leitor evolui)]

$\mathrm{PN} 15=\mathrm{F}\left[\mathrm{S}_{1}\right.$ saber $\rightarrow\left(\mathrm{S}_{2}\right.$ leitura $\wedge \mathrm{O}_{\mathrm{v}}$ interage com a cultura e os esquemas dominantes de um meio e de uma época)]

$\mathrm{PN16}=\mathrm{F}\left[\mathrm{S}_{1}\right.$ saber $\rightarrow\left(\mathrm{S}_{2}\right.$ leitura $\wedge \mathrm{O}_{v}$ afirma sua dimensão simbólica agindo nos modelos do imaginário coletivo quer os recuse quer os aceite)]

Quadro IV. Leitura: processo simbólico

O discurso pragmático analisado, por meio dos programas narrativos, revela que houve uma conjunção com o conhecimento ou ideia de leitura desenvolvida pelo autor. Este não demostra como foi sua produção do saber, mas o resultado desse conhecimento, por isso, as relações dos elementos do discurso foram esta- belecidas por pressuposição. $O$ conhecimento descrito se assemelha à descrição de conceitos expressos em termos no texto.

A dimensão do discurso cognitivo apresenta o reconhecimento da transformação de estado desse saber. $O$ autor descreveu no texto, a partir de uma questão, um estado inicial de nãosaber o que é a leitura e ao descrevê-la, passa para o estado final de saber algo sobre a leitura. Portanto, o tema principal de nível profundo pode ser composto principalmente pelo termo "leitura", o que demonstra ser o aboutness do texto. O nível profundo apresenta o tema principal ou aboutness "Não saber sobre leitura versus Saber sobre leitura", o que representa o tema em dimensão cognitiva e pragmática.

A análise realizada do texto científico demonstra que é possível identificar o aboutness, considerando as dimensões pragmática e cognitiva, que são complementares. Consequentemente, tornou possível a identificação do aboutness em nível profundo, tal como ocorre em Análise Documental de Conteúdo com o texto narrativo de ficção. Mas, considerando para isso o termo explícito como tema principal. Por isso, esta análise elucidou a metodologia de identificação do aboutness em discurso científico por meio das regras de construção do saber da Semiótica Greimasiana e Percurso Gerativo de Sentido em Ciência da Informação.

\section{Considerações finais}

A Semiótica Greimasiana explica a análise do discurso científico e a produção de seu sentido por meio do entendimento das relações das dimensões pragmática e cognitiva, entre outros fatores. Assim, demonstra que um discurso científico descreve o estado inicial de um saber (ideia ou conhecimento sobre algo), sua transformação e seu estado final.

A análise realizada do texto científico de Jouve (2002) permitiu verificar que é possível a identificação do tema principal de nível profundo ou aboutness, tal como ocorre em textos narrativos de ficção em Análise Documental de Conteúdo, considerando também o Percurso Gerativo de Sentido. Mas, com a particularidade de que o termo principal ("leitura") é apresentado de forma explícita devido ao tipo de discurso.

Também, permitiu elucidar alguns elementos do discurso científico importantes para a compreensão e identificação de seu sentido e aboutness, mostrando o desenvolvimento do saber sobre os conceitos descritos nos textos. Por isso, contribui para a Análise Documental de Conteúdo no entendimento sobre o desen- 
volvimento desse saber em textos científicos e sua relação com o aboutness.

Espera-se também que esta análise possa oportunizar novas pesquisas que demonstrem a análise do aboutness também sobre textos mais complexos, ou seja, com temas múltiplos, conceitos complexos, com diferentes métodos e organizações de estruturas textuais.

\section{Referências}

Alves, R. C. V. (2008). Análise documental de textos literários infanto-juvenis. Marília: Unesp. Dissertação Mestrado Ciência da Informação. 2008.

Antonio, D. M.; Moraes, J.B.E.de; (2010). Análisis documental de obras de ficción: aspectos metodológicos y de aplicabilidad. // Scire. (2010) 71-78

Barros, D.L.P.de (2008). Teoria semiótica do texto. São Paulo: Ática, 2008.

Beghtol, C. (1986). Bibliographic classification theory and text linguistics. // Journal of Documentation. 42:2 (june, 1986) 84-113.

Fiorin, J. L. (1999). Sendas e veredas da semiótica narrativa e discursiva. // Delta. 15:1 (fev./jul. 1999). http://www. scielo.br/scielo.php?script=sci_arttext\&pid=S0102-44501 999000100009\&lng=en\&nrm=iso (2006-10-28).

Fiorin, J. L. (2008). Elementos de análise do discurso. São Paulo: Contexto, 2008
Gardin, J. C.; et al.(1981). La logique du plausible. Paris: Maison des Sciences de l'Homme, 1981.

Greimas, A. J. (1973) Semântica estrutural. São Paulo: Cultrix, 1973.

Greimas, A. J. (1976) Semiótica do discurso científico: da modalidade. São Paulo: Difel, 1976.

Greimas, A. J. (1979). Acidentes nas ciências ditas humanas. // Greimas, A. J.; Landowski, E. Análise do discurso em Ciências Sociais. São Paulo: Global, 1979.

Greimas, A. J.; Courtés, J. (2008). Dicionário de Semiótica. São Paulo: Contexto, 2008.

Greimas, A. J.,Landowski, E. (1979). Os percursos do saber. // Greimas, A. J.; Landowski, E. Análise do discurso em Ciências Sociais. São Paulo: Global, 1979.

Guimarães, J. A. C.; Moraes, J. B. E. de; Guarido, M. D. M. (2007). Análisis documental de contenido de textos narrativos // Ibersid. 1 (2007) 93-100.

Jouve, V. (2002) A leitura. São Paulo: Unesp, 2002.

Moraes, J.B.E.de (2011). A questão do aboutness no texto narrativo de ficção. Marília: Unesp. Tese Livre Docência Linguística e Documentação. 2011.

Moraes, J. B. E.de; Damazo, A.; Lara, L. M. de. (2008). Avaliação da proposta de análise documental de textos narrativos de ficção. // Ibersid. (2008) 185-190.

Tatit, L. (2003). Abordagem do texto. // Fiorin, J. L.(org.). Introdução à Linguística I. São Paulo: Contexto, 2003. 187-209.

Enviado: 2014-04-28. Segunda versión: 2014-06-10. Aceptado: 2013-08-19. 
